\title{
Quantity and quality of potential drug interactions with coumarin anticoagulants in the Netherlands
}

\author{
Fernie J. A. Penning-van Beest · Jeroen Koerselman • \\ Ron M. C. Herings
}

Received: 16 November 2006/Accepted: 25 March 2007/Published online: 24 April 2007

(C) Springer Science+Business Media B.V. 2007

\begin{abstract}
Objective Coumarin anticoagulants are prone to potentially life-threatening drug-drug interactions due to a combination of unfavorable properties. However, real life data on the actual occurrence are scarce. The aim of this study was to quantify and qualify potential drug interactions with coumarin anticoagulants in daily practice.

Methods A cohort study including all users of phenprocoumon or acenocoumarol during the period 1991-2003 in the PHARMO Record Linkage System. All 24 individual drugs and 11 drug groups interacting with coumarins according to central database used in the Dutch pharmacies were considered.

Main outcome measure Frequency and type of potential drug interactions during anticoagulant therapy with coumarins.

Results $\quad 48,627$ out of 76,455 mainly acenocoumarol-users (64\%) were dispensed at least one potentially interacting drug (PID) during anticoagulant therapy. About $35 \%$ of these cases were dispensed a (very) strongly interacting drug, whereas $3 \%$ were dispensed a contraindicated drug. Antibacterial drugs and NSAIDs (39\% and 37\% of all users, respectively) were the most frequently dispensed PIDs.

Conclusion Potential drug interactions with coumarins frequently occur in daily practice, confronting two-thirds of
\end{abstract}

F. J. A. Penning-van Beest $(\bowtie) \cdot$ J. Koerselman .

R. M. C. Herings

PHARMO Institute, P.O. Box 85222, 3508 AE Utrecht,

The Netherlands

e-mail: fernie.penning@pharmo.nl

R. M. C. Herings

Department of Pharmaco-epidemiology and Pharmacotherapy,

Utrecht Institute for Pharmaceutical Sciences, Utrecht,

The Netherlands patients with an increased risk of bleeding. To a large part, this is attributable to commonly prescribed medication like antibacterial drugs and NSAIDs. This situation substantiates the need for proper monitoring or new anticoagulants with less drug-drug interactions.

Keywords Acenocoumarol - Antibacterial drugs . Anticoagulants · Coumarins - Drug-Drug interactions . NSAIDs · Phenprocoumon · The Netherlands

\section{Impact of findings on practice}

- Potential drug interactions occur in two-thirds of patients using coumarins.

- Pincipal interactions concern the commonly prescribed anti-bacterial drugs and NSAIDs.

- The high potential for drug interactions of coumarins puts patients at increased risk of bleeding, and costs time and Money.

\section{Introduction}

Coumarin anticoagulants (i.e. acenocoumarol, phenprocoumon and warfarin) are extensively used for the treatment and long-term prevention of thromboembolic diseases [1]. These drugs induce anticoagulation by antagonizing vitamin $\mathrm{K}$, thereby impairing the biologic activity of the vitamin- $\mathrm{K}$ dependent coagulation factors (factor II, VII, IX, and X) [2, 3]. Apart from their benefit, coumarin anticoagulants are prone to potentially life threatening drug-drug interactions due to: (i) high protein 
binding; (ii) cytochrome P450 dependent metabolism; and (iii) a narrow therapeutic range $[2,4]$.

In the Netherlands, a routine screening for potential interactions is implemented in all pharmacies. Before drugs are dispensed to the patients, potential interactions have to be detected by the automated computer system. The entire list of drugs which are supposed to interact with coumarins comprises about 200 different compounds (Z-Index BV, The Hague, The Netherlands, URL: http://www.z-index.nl). Some of these potentially interacting drugs (PIDs) are not allowed to be dispensed to the patient, but should be substituted with another drug. However, the majority of the PIDs can be normally dispensed to the patient after the anticoagulation clinic and/or the patient have been informed (Dutch standard on coumarin interactions; available through the internet via URL: http://www.fnt.nl).

Patients on coumarins who are dispensed a PID, are confronted with an increased risk of bleeding or thromboembolism. In addition, the effectiveness of the interacting drug may be hampered.

\section{Aim of the study}

To date, "real life" data on the occurrence of potential drug interactions with coumarins are scarce [5]. Therefore, we performed a cohort study to quantify and qualify potential drug interactions with coumarin anticoagulants in daily practice.

\section{Methods}

\section{Setting}

Data were obtained from the PHARMO Record Linkage System, which includes, among other databases, the drug-dispensing records from community pharmacies and hospital discharge records of more than 1 million community-dwelling inhabitants of 40 demographically defined areas in The Netherlands. For all residents, the computerized drug-dispensing histories contain data concerning the dispensed drug, type of prescriber, dispensing date, dispensed amount, prescribed dose regimens, and the legend duration of use (prescription length). All drugs are coded according to the Anatomical Therapeutic Chemical (ATC) Classification. The hospital records include detailed information concerning the primary and secondary diagnoses, procedures, and dates of hospital admission and discharge. All diagnoses are coded according to the International Classification of Diseases, Ninth Revision, Clinical Modification (ICD-9-CM). For a detailed description of the database, which is representative for the Dutch population, we refer to previous work [6].
Study cohort

The study population included all users of phenprocoumon (ATC-code B01AA04) or acenocoumarol (ATC-code B01AA07) in the period 1991-2003. No other coumarins are available at the Dutch market. All patients were followed from the first dispensing in the period 1991-2003 until the last dispensing, or the end of follow-up (October 1, 2004), whichever event was earliest.

\section{Potential drug-drug interactions}

All 24 individual drugs and 11 drug groups interacting with coumarins according to the current $Z$-index database, the central database used in the Dutch pharmacies (Z-Index BV, The Hague, The Netherlands, URL: http://www. z-index.nl), were considered for this study. For all PIDs, relevance was classified into five categories according to the Dutch standard on coumarin interactions (available through the internet via URL: http://www.fnt.nl). Category 1 included all contraindicated drugs that very strongly interact with coumarins and may not be used together with coumarins. Category 2 comprised all very strongly interacting drugs for which a substitute is lacking and that necessitate intensive monitoring of anticoagulation. Category 3 included all strongly interacting drugs that preferably are substituted or otherwise necessitate intensive monitoring of anticoagulation. Category 4 encompassed all drugs that moderately or indistinctly interact with coumarins. Category 5 included all drugs that do not influence the intensity of the anticoagulant effect of coumarin anticoagulants, but increase the risk of bleeding by interfering with hemostasis or by their ulcerogenic effect.

To study the frequency of potential drug interactions during anticoagulant therapy with coumarins, treatment episodes of coumarins during follow-up were established for each patient, based on the method of Catalan [7]. In general, a treatment episode is defined as a period of time in which a continuous specific pharmacotherapeutic treatment takes place. Coumarin treatment was considered to be uninterrupted if the gap between the start of two consecutive dispensings was less than 180 days. A treatment episode was measured as the time span between the starting date of the first dispensing and the expiry date of the final dispensing. The latter was set at the average duration time after the last dispensing date. Patients may have had more than one treatment episode of coumarins.

For each PID and PID group, the number of dispensings during a coumarin treatment episode and the number of patients involved was assessed. For frequently occurring PID groups, the individual PIDs within this group were determined. For all patients involved in at least one potential drug-drug interaction (the "cases"), the number 
of different PIDs and the relevance of the exposed potential interaction(s) were determined.

\section{Results}

The study cohort included 76,455 users of mainly acenocoumarol in the period 1991-2003 (Table 1). Gender was equally divided, and about two-thirds of the coumarinusers were older than 60 years of age. A quarter of the users had more than 730 days of coumarin treatment during follow-up.

In total, 48,627 patients or "cases" (64\% of all coumarin-users) were involved in at least one potential drug interaction during anticoagulant therapy. Gender was equally divided, and $75 \%$ of the cases was older than 60 years of age (Table 2). Multiple potential drug interactions occurred in more than half of the cases; $5 \%$ of the cases were even dispensed eight or more different PIDs. About $35 \%$ of the cases were dispensed a (very) strongly interacting drug, that necessitates intensive monitoring of anticoagulation (relevance category 2 and 3 ). A contraindicated drug (relevance category 1) was dispensed in $3 \%$ of the cases.

The most frequently dispensed PIDs during anticoagulant therapy with coumarins were antibacterial drugs and non-steroidal anti-inflammatory drugs (NSAIDs), encompassing nearly 120,000 antibacterial dispensings in $39 \%$ of

Table 1 Characteristics of users of acenocoumarol or phenprocoumon in the period 1991-2003

\begin{tabular}{llrl}
\hline Characteristic & & $\begin{array}{l}\text { Number } \\
(N=76,455)\end{array}$ & $\begin{array}{l}\text { Percentage } \\
\text { of } \\
\text { total }(\%)\end{array}$ \\
\hline Gender & Men & 37,767 & 49.4 \\
& Women & 38,688 & 50.6 \\
Age at start follow-up & $<20$ & 757 & 1.0 \\
(in years) & $20-44$ & 8,659 & 11.3 \\
& $45-59$ & 14,343 & 18.8 \\
& $60-74$ & 30,132 & 39.4 \\
Type of coumarin at & Acenocoumarol & 68,519 & 89.6 \\
start follow-up & Phenprocoumon & 7,936 & 10.4 \\
Number of coumarin & 1 & 55,388 & 72.5 \\
treatment episodes & 2 & 11,958 & 15.6 \\
during follow-up & $\geq 3$ & 9,109 & 11.9 \\
Number of coumarin & $\leq 90$ & 26,658 & 34.8 \\
treatment days & $91-180$ & 11,253 & 14.7 \\
during follow-up & $181-365$ & 9,094 & 11.9 \\
& $366-729$ & 8,832 & 11.6 \\
& $\geq 730$ & 20,618 & 27.0 \\
\hline
\end{tabular}

Table 2 Demographics and relevance of potential drug interactions during anticoagulant therapy with coumarins in the period 1991-2004

\begin{tabular}{|c|c|c|c|}
\hline Characteristic & & $\begin{array}{l}\text { Number of patients } \\
(\mathrm{N}=48,627)\end{array}$ & $\begin{array}{l}\text { Percentage } \\
\text { of } \\
\text { total }(\%)\end{array}$ \\
\hline \multirow[t]{2}{*}{ Gender } & Men & 24,052 & 49.5 \\
\hline & Women & 24,575 & 50.5 \\
\hline \multirow{5}{*}{$\begin{array}{l}\text { Age at start PID } \\
\text { (in years) }\end{array}$} & $<20$ & 242 & 0.5 \\
\hline & $20-44$ & 3,661 & 7.5 \\
\hline & $45-59$ & 8,044 & 16.5 \\
\hline & $60-74$ & 19,135 & 39.4 \\
\hline & $>74$ & 17,545 & 36.1 \\
\hline \multirow[t]{4}{*}{ Number of PIDs } & 1 & 20,260 & 41.7 \\
\hline & $2-4$ & 20,788 & 42.7 \\
\hline & $5-7$ & 5,306 & 10.9 \\
\hline & $\geq 8$ & 2,273 & 4.7 \\
\hline \multirow[t]{5}{*}{ Relevance $^{\mathrm{a}}$} & Category 1 & 1,574 & 3.2 \\
\hline & Category 2 & 1,476 & 3.0 \\
\hline & Category 3 & 14,609 & 30.0 \\
\hline & Category 4 & 31,846 & 65.5 \\
\hline & Category 5 & 28,062 & 57.7 \\
\hline
\end{tabular}

a Numbers do not add up to $100 \%$ as patients may have been involved in multiple PIDs of different relevance

PID: potentially interacting drug

all coumarin-users, and almost 127,000 NSAID-dispensings in $37 \%$ of all coumarin-users, respectively (Table 3 ). The top three potentially interacting antibacterial drugs were amoxicillin, doxycycline, and amoxicillin plus clavulanic acid (dispensed to 13,13, and 10\% of all coumarin-users, respectively). Diclofenac, ibuprofen, and naproxen were the three most frequently dispensed NSAIDs (dispensed to 15, 9 , and $7 \%$ of all coumarin-users, respectively).

\section{Discussion}

The results of the present population-based cohort study show that potential drug interactions with coumarins frequently occur in daily practice. About two-thirds of users of acenocoumarol or phenprocoumon (50,000 out of 76,000 users) were dispensed at least one PID. In about one third of these cases, this concerned (very) strongly interacting drugs. The most frequently dispensed PIDs were NSAIDs.

Although, routine screening for potential interactions is implemented at all pharmacies in the Netherlands, a pharmacist may selectively switch off one or more of the interaction-modules. Consequently, some potential drugdrug interactions may remain undetected. This may explain the dispensing of contraindicated drugs (category 1 PIDs) to $3 \%$ of the cases and possibly part of the preferably 
Table 3 Frequency of potential drug interactions during anticoagulant therapy with coumarins in the period 1991-2004

\begin{tabular}{|c|c|c|c|c|c|}
\hline \multirow[t]{2}{*}{ PID (group)* } & \multirow[t]{2}{*}{ ATC-code(s) } & \multirow[t]{2}{*}{$\begin{array}{l}\text { Relevance } \\
\text { category }\end{array}$} & \multirow[t]{2}{*}{$\begin{array}{l}\text { Number of } \\
\text { dispensings }\end{array}$} & \multicolumn{2}{|c|}{$\begin{array}{l}\text { Number of } \\
\text { patients } \\
(N=76,455)\end{array}$} \\
\hline & & & & $n$ & $\%$ \\
\hline Antibacterial drugs ${ }^{\mathrm{a}}$ & J01, A07AA & 4 & 119,517 & 30,022 & 39.3 \\
\hline NSAIDs ${ }^{b}$ & $\begin{array}{l}\text { M01A, C01EB03, N02BB02, N02BB04, N02BE51, N02BA, } \\
\text { B01AC06, B01AC08, B01AC30 }\end{array}$ & 5 & 126,593 & 28,364 & 37.1 \\
\hline Miconazole & A07AC01, G01AF04 & 1 & 1,706 & 890 & 1.2 \\
\hline Amiodarone & C01BD01 & 3 & 35,387 & 4,238 & 5.5 \\
\hline Cotrimoxazole & J01EE01, J01EE03 & 3 & 7,189 & 4,087 & 5.3 \\
\hline SSRIs & N06AB & 4 & 31,078 & 3,380 & 4.4 \\
\hline Thyreomimetics & $\mathrm{H} 03 \mathrm{~A}$ & 3 & 24,052 & 2,323 & 3.0 \\
\hline Allopurinol & M04AA01 & 3 & 15,943 & 1,742 & 2.3 \\
\hline $\begin{array}{l}\text { Anti-epileptics (enzyme } \\
\text { inducing) }\end{array}$ & N03AA, N03AB02, N03AF01, N05CA, N05CB02, L02BG01 & 2 & 16,206 & 1,414 & 1.8 \\
\hline Cimetidine $^{c}$ & A02BA01 & 3 & 5,152 & 931 & 1.2 \\
\hline Fibrates & $\mathrm{C} 10 \mathrm{AB}$ & 3 & 8,228 & 901 & 1.2 \\
\hline Metronidazole & J01XD01,G01AF01, P01AB01 & 3 & 1,246 & 768 & 1.0 \\
\hline Thyreostatics & H03B & 3 & 7,125 & 751 & 1.0 \\
\hline
\end{tabular}

* The following PIDs or PID-groups each concerned less than 1.0\% of the patients: Androgens (A14A,G03B,G03XA01), Azapropazone (M01AX04), Azathioprine (L04AX01), Benzbromarone (M04AB03), Bile acid sequestrants (C10AC), Disopyramid (C01BA03), Disulfiram (N07BB01), Fluconazole (J02AC01-except for single dosages), Griseofulvin (D01BA01), Isoniazid (J04AC01), Itraconazole (J02AC02), Ketoconazole (J02AB02), Mercaptopurine (L01BB02), Non-nucleoside reverse transcriptase inhibitors (J05AG), Phenylbutazone (M01AA01), Propafenone (C01BC03), Protease inhibitors (J05AE), Quinidine (C01BA01), Rifampicin (J04AB02,J04AM02), Rifabutin (J04AB04), Tamoxifen (L02BA01), and Voriconazole (J02AC03)

${ }^{a}$ exclusive cotrimoxazole and metronidazole; ${ }^{b}$ Exclusive azapropazone and phenylbutazone; ${ }^{c}$ Applies to acenocoumarol only

ATC-code: Anatomical Therapeutic Chemical Classification System - code, WHO Collaborating Centre for Drug Statistics Methodology, ATC/ DDD index 2005 (URL: http://www.whocc.no/atcddd)

NSAID: non-steroidal anti-inflammatory drug; PID: potentially interacting drug; SSRI: selective serotonin reuptake inhibitor

substituted category 3 PIDs dispensed to $30 \%$ of the cases. Category 3 PIDs may not be avoidable due to, for example, certain patient factors or comorbidity, or lack of prescription alternatives. Unfortunately, we did not have this information. An improved use of the routine screening may reduce the number of PIDs dispensed. It is yet to be expected that this reduction will only be limited.

The study encompassed the period 1991-2003. As mentioned in the methods, the PIDs considered for the study were based on the current interaction database at the time the study was performed (i.e., the 2004 version). The majority of the selected PIDs were already included in this interaction database before 1991 and were available during the whole study period. However, eight out of 24 selected individual drugs (e.g. benzbromarone and disopyramid) and three out of 11 selected drug groups (non-nucleoside reverse transcriptase inhibitors, protease inhibitors and thyreostatics) were included in the interaction database from 1998 or later, and so were only available during the second half of the study period. Consequently, the number of dispensings and patients will be smaller for these drugs. However, our finding that antibacterial drugs and NSAIDs are the most frequently dispensed PIDs is still valid; the number of patients for these two PIDs is much higher than for the other PIDs.

The type of coumarin mainly used by our study cohort was acenocoumarol. In many countries, warfarin is the coumarin of first choice. Potential drug interactions during anticoagulant therapy with warfarin have been shown to be a frequent issue, as well [8,9]. Among 134,833 patients receiving long-term warfarin therapy, 109,998 (81.6\%) were prescribed a concurrent prescription for at least one PID [8].

Given the resemblance in the metabolism of acenocoumarol and warfarin, both types of coumarin are likely to be sensitive to the same PIDs. However, as the CYP2C9 isoenzyme appears to be less important for the clearance of acenocoumarol than for the clearance of warfarin, acenocoumarol may be less sensitive than warfarin [10]. Consequently, the outcome of a drug interaction may be different for acenocoumarol and warfarin.

Patients on coumarins, who are dispensed a PID, are exposed to an increased risk of, mainly, bleeding, or thromboembolism [4]. Bleeding is the most common 
complication of anticoagulant therapy. Major or lifethreatening hemorrhage during anticoagulant therapy with coumarins has been shown to occur at an estimated rate of 1.1-3.6 per 100 patient-years [11-13]. Concomitant use of other drugs is one of the major determinants of bleeding during anticoagulant therapy [14]. In the present study, antibacterial drugs and NSAIDs were the main PIDs dispensed during coumarin therapy. Antibacterial drugs enhance the anticoagulant effect of coumarins, thereby increasing the risk of bleeding [15]. In a population-based cohort study by Visser et al. use of antibacterial drugs was associated with a 4-20 times increased risk of over anticoagulation [16]. The risk of major bleeding associated with the use of antibacterial drugs, has been shown to be four to seven times increased [17]. NSAIDs and antithrombotic salicylates mainly increase the risk of bleeding by interfering with hemostasis by inhibiting platelet function, or by their ulcerogenic effect [18]. In a previous study, we found a 2-7 times increased risk of major bleeding with the use of NSAIDs during anticoagulant therapy [17]. Although, the risks of major bleeding increase quite strongly in case of concomitant use of antibacterial drugs and NSAIDs, absolute incidence rates are low. Regarding antibacterial drugs an incidence rate of 4.3-7.4 major bleedings per 100 patient-years has been reported. For NSAIDs this rate was $2.4-7.4$ per 100 patient-years [17].

In addition to putting patients at an increased risk, the high drug interaction potential of coumarins costs time and money. If at the pharmacy, potential interactions are detected by the automated computer system, actions have to be taken before drugs are dispensed. The necessary action ranges from just informing the anticoagulation clinic and/or the patient, to consulting the prescribing physician for a substitute (Dutch standard on coumarin interactions; available through the internet via URL: http://www.fnt.nl). In addition, the use of a PID may necessitate intensive monitoring by the anticoagulation clinic. This concerned one-third of our cases.

\section{Conclusion}

The results of the present population-based cohort study show that potential drug interactions during anticoagulant therapy with coumarins frequently occur in daily practice, confronting about two-third of patients with an increased risk of, mainly, bleeding. To a large part, this is attributable to commonly prescribed medication like antibacterial drugs and NSAIDs. This situation substantiates the need for new anticoagulants with less drug-drug interactions.

Acknowledgment This study was financially supported by an unrestricted grant from AstraZeneca, Mölndal, Sweden.

\section{References}

1. British Committee for Standards in Haemotology. Guidelines on oral anticoagulation. 3rd ed. Br J Haematol 1998;101(2):374-87.

2. Hirsh J, Dalen J, Anderson DR, Poller L, Bussey H, Ansell J, et al. Oral anticoagulants: mechanism of action, clinical effectiveness, and optimal therapeutic range. Chest 2001;119 (1 Suppl):8S-21S.

3. Sadowski JA, Booth SL, Mann KG, Malhotra OP, Bovill EG. Structure and mechanism of activation of vitamin $\mathrm{K}$ antagonists. London: Arnold; 1996.

4. Harder S, Thurmann P. Clinically important drug interactions with anticoagulants. An update. Clin Pharmacokinet 1996;30(6):416-44.

5. Holbrook AM, Pereira JA, Labiris R, McDonald H, Douketis JD, Crowther M, et al. Systematic overview of warfarin and its drug and food interactions. Arch Intern Med 2005;165(10):1095-106.

6. Herings RMC. PHARMO: a record linkage system for postmarketing surveillance of prescription drugs in The Netherlands. Thesis. Utrecht: Utrecht University; 1993.

7. Catalan VS, Lelorier J. Predictors of long-term persistence on statins in a subsidized clinical population. Value Health 2000;3(6):417-26.

8. Wittkowsky AK, Boccuzzi SJ, Wogen J, Wygant G, Patel P, Hauch O. Frequency of concurrent use of warfarin with potentially interacting drugs. Pharmacotherapy 2004;24(12):1668-74.

9. Howard PA, Ellerbeck EF, Engelman KK, Patterson KL. The nature and frequency of potential warfarin drug interactions that increase the risk of bleeding in patients with atrial fibrillation. Pharmacoepidemiol Drug Saf 2002;11(7):569-76.

10. Ufer M. Comparative pharmacokinetics of vitamin k antagonists: warfarin, phenprocoumon and acenocoumarol. Clin Pharmacokinet 2005;44(12):1227-46.

11. Palareti G, Leali N, Coccheri S, Poggi M, Manotti C, D'Angelo A, et al. Bleeding complications of oral anticoagulant treatment: an inception-cohort, prospective collaborative study (ISCOAT). Italian study on complications of oral anticoagulant therapy. Lancet 1996;348(9025):423-8.

12. van der Meer FJ, Rosendaal FR, Vandenbroucke JP, Briet E. Assessment of a bleeding risk index in two cohorts of patients treated with oral anticoagulants. Thromb Haemost 1996;76(1):12-6.

13. Landefeld CS, Beyth RJ. Anticoagulant-related bleeding: clinical epidemiology, prediction, and prevention. Am J Med 1993;95(3):315-28.

14. Levine MN, Raskob G, Landefeld S, Kearon C. Hemorrhagic complications of anticoagulant treatment. Chest 2001;119 (1 Suppl):108S-121S.

15. Stockley IH. Drug interactions. 6th ed. London: The Pharmaceutical Press; 2002.

16. Visser LE, Penning-van Beest FJ, Kasbergen AA, De Smet PA, Vulto AG, Hofman A, et al. Overanticoagulation associated with combined use of antibacterial drugs and acenocoumarol or phenprocoumon anticoagulants. Thromb Haemost 2002;88(5):705-10.

17. Penning-van Beest F, Erkens J, Petersen KU, Koelz HR, Herings R. Main comedications associated with major bleeding during anticoagulant therapy with coumarins. Eur J Clin Pharmacol 2005;61(5-6):439-44.

18. Majerus PW, Tollefsen DM. Anticoagulant, thrombolytic, and antiplatelet drugs. In: Hardman JG, Goodman Gilman A, Limbird LE editors. The pharmacological basis of therapeutics. 10th ed. New York: McGraw-Hill; 2001. 